\title{
Risk Mapping in the Selected Town
}

\author{
ALŽBĚTA ZÁBRANSKÁ, JAKUB RAK, PETR SVOBODA \\ Tomas Bata University in Zlín \\ Nám. T. G. Masaryka 5555, 76001 \\ CZECH REPUBLIC \\ a_zabranska@utb.cz,jrak@utb.cz,psvoboda@utb.cz
}

\begin{abstract}
This article describes the risk analysis and method of risk mapping in the town of Uherský Brod invented by the Fire Rescue Service of the Moravian-Silesian Region on the basis of the methodology recommended by the European Union. QGIS software tool was used for risk mapping in order to visualize the maps. The risk analysis was performed on the basis of identification of types of hazards that are very likely to occur in Uherský Brod. Subsequently, the results of the risk analysis were processed by means of the software QGIS. This software enables the creation of the risk map once the individual maps have been compiled. Evaluating the resulting risk map, the individual areas of the town were assessed according to the discovered level of risk. Risk maps can consequently serve.
\end{abstract}

Key-Words: - Emergency event, hazard, geographic information system, modeling, risk analysis, risk mapping.

\section{Introduction}

The development of social society as such has always been accompanied by improvement in its protection. In the past mankind used to feel threatened by emergency events of natural character (floods, earthquakes or extreme meteorological phenomena). Eventually, with the continuous improvement of modern technologies the emergency events of anthropogenic character (industrial and traffic accidents) began to emerge. Society has adopted a set of specific measures, which determine the level of preparedness, to overcome the emergency events. In order for these measures to be effective and purposive, it is primarily necessary, among other things, to perform a quality risk analysis. On the basis of the evaluation of the analysis, it is possible to identify the overall weight of risks in the given territory and to define parameters for determining the level of preparedness.

The essence of the risk mapping method is elaborated on in a handbook prepared under the European project Interreg IIIC SIPROCI, which aims to improve the response to emergency events using the interregional cooperation of European countries. [1]

\section{Problem Formulation}

All objects, phenomena, risks or activities are related to a certain space and can interact with each other. For this reason, it is necessary to know individual connections that could cause this influence together with the relevant spatial information. This makes it possible to mark out floodplains, locate sources of dangerous chemical substances, or plan a route from $\mathrm{X}$ to $\mathrm{Y}$. [7]

Safety risks have their spatial information, which makes it possible to identify risk areas in the analyzed territory. However, it is important to consider the key aspect, which is the changeable intensity of most types of hazards in the given territory. As a rule, the danger is more intense directly in the risk area adjacent to the source of danger compared to more distant areas. The primary support for risk mapping was provided by the geographic information system (GIS). [3] [5]

For the purposes of this paper the multi-platform geographic information system QGIS was used.

In the Czech Republic the risks differ, the specific type of risk is characteristic by the territory and the intensity it manifests itself with. The risk of an uncontrolled rising of a firedamp to the ground in the Moravian-Silesian region can serve as an example, while in the Zlín region its occurrence is only incidental. In risk mapping, a particular type of hazard must be expressed by a cartographic projection, in other words, it must have a specific territorial manifestation. [5]

According to statistical data of the analyzed territory of the town of Uherský Brod, the following emergency events may occur: natural flood, extraordinary flood, leakage of a dangerous chemical substance from a stationary source, leakage of a dangerous chemical substance during transport, railway and traffic accidents, damage to the slope stability, fire, snow calamity and bird flu. 
Risk mapping is a process during which areas with different levels of risk are identified. This includes results of risk assessments depicted on risk maps. The risk map represents the level of damage, which can be expected in the given territory. It also allows identification of composition and level of risk for each part of the analyzed area. Nevertheless, the main condition is the inclusion of only such emergency events whose manifestation can be expressed by means of a cartographic projection. [1]

\section{Problem Solution}

Altogether, the risk mapping includes 5 fundamental phases: determining the level of risk (a map of hazard), vulnerability assessment (a vulnerability map), cumulative risk assessment (a map of cumulative risk), preparedness assessment (a preparedness map), an accumulated risk assessment (an accumulated risk map). [1]

Only the first three phases will be discussed in this article.

\subsection{Materials and Methods}

The risk mapping method is a process during which areas with different levels of risk are identified. Mapping involves the interaction of different types of hazards identifying the vulnerability of the area and level of preparedness of the analyzed area. Risk mapping is implemented based on the geographic information system "GIS" (in this case Quantum GIS) and statistical and numerical analyzes. [1]

Quantum GIS ("QGIS") is a multiplatform geographic information system which in particular allows to view, create and edit raster and vector geodata, and to create map outputs. It was developed as an Open Source, which guarantees a long-term sustainability of the invented method of operation and its extensibility. [2][3]

In order to view individual manifestations of emergency events in the map, layers or data from which the layer can be generated in QGIS must exist. This includes, for instance, a list of roads, watercourses, or objects with specific address. For the purposes of the article, numerical model calculations for processing the manifestations of individual emergency events (e.g. leakage of dangerous chemical substances-the ALOHA simulation software), long-term statistical monitoring of the weather or natural phenomena (especially landslides) were used. [1] [5]

In addition, the following maps and databases were used for the needs of risk mapping: maps of flood risks, maps of areas affected by snow, digital geographic model of the Czech Republic ZABAGED with GIS map layers, which contain sub-categories of vulnerability and therefore they can be used for cartographic projection of vulnerability. Also, the OpenStreetMap - a vector map database that is freely accessible on the Internet-was used. [4] [8]

In order to determine the value of the risk level a multi-criteria analysis using the method of expert estimation was used. This analysis was performed on the basis of statistical data on the occurrence of emergency events in the last 20 years in the territory of Uherský Brod.

An essential document for addressing this issue is the Risk Mapping Methodology invented by the Fire Rescue Service of the Moravian-Silesian Region. [1]

During the risk mapping process, the issues of risk mapping were consulted with experts in crisis and emergency planning.

\subsection{Problem Solution}

For the needs of risk mapping, a multi-criteria analysis with the expert estimation invented by the Fire Rescue Service of the Moravian-Silesian Region in 2002 was used. This method is based on the estimation of the values of the criteria for the individual types of hazards, including the effect of possible consequential emergency events, and it also determines a level of risk in order to compare types of emergency events. This method of risk analysis was performed on the basis of statistical data on emergency events in the analyzed territory in the town of Uherský Brod, which occurred in the last 20 years.

Within a multi-criteria analysis, the risk represents: "expected negative consequences due to activation of danger in the given territory." [1]

In the risk mapping method the risk level is understood as: "the value of probability of occurrence of negative consequences during the given type of emergency event." [1]

$$
M R=F \times N
$$

Where:

$F$ - the frequency of possible occurrence of the emergency event (EE) for a particular type of hazard.

$N$-consequences of the EE. 
The consequences of the $\mathrm{EE}(N)$ can be further expressed as:

$$
N=\frac{K t \times K o h r \times K i z s}{P r}
$$

Where:

$K t$ - the coefficient of the expected duration of the emergency event,

$K o h r$ - the coefficient of a threat during the EE,

Kizs - the coefficient expressing the need for the forces and resources of the Integrated Rescue System and the need for coordination of the EE,

$\mathrm{Pr}$ - the coefficient of possible time prediction.

The threat coefficient (Kohr) is given by the sum of individual elements of different weightings. In order to express the different weighting of individual elements of the threat, weight coefficients are introduced into the calculation. [1]

$K o h r=(K o x V K o)+(K p x V K p)+(K e x V k e)+$

$(K b \times V K b)+(K z \times V K z)+(K d+V K d)$

Where:

$K o$ - the coefficient of individual elements of threat,

$K p$ - the coefficient of individual elements of the affected area,

$\mathrm{Ke}$ - the coefficient of threat to biotic environment,

$K b$ - the coefficient of threat to buildings and built-up areas,

$K z$ - the coefficient of threat to breeding animals,

$K d$ - the coefficient of interruption of traffic,

$V K o, V K p \ldots$ individual weight coefficients.

The coefficient of the Integrated Rescue System (Kizs) consists of coefficients of the Itegrated Rescue System (IRS) sub-elements. [1]

$$
K i z s=(K s \times V K s)+(K k \times V K k)
$$

Where:

$K s$ - the coefficient of the need for forces and resources of the IRS,

$K k$ - the coefficient of the need to coordinate the emergency event,

$V K s, V K k$ - weight coefficients.

The weight coefficients take into consideration the weighting of individual elements. The values of weight coefficients and the scale for their values are based on the Risk Mapping Handbook. The table below represents the final levels of risk for individual emergency events that may occur in the analyzed territory of Uherský Brod. [1]

The table below represents the final levels of risk for individual emergency events that may occur in the analyzed territory of Uherský Brod.

\begin{tabular}{|l|c|}
\hline Type of danger & $\begin{array}{c}\text { Risk } \\
\text { level }\end{array}$ \\
\hline Natural flood & 268,704 \\
\hline$Q 20$ - 730 persons & 247,968 \\
\hline$Q 50$ - 1400 persons & 302,4 \\
\hline$Q 100$ - 1 840 persons & 255,744 \\
\hline $\begin{array}{l}\text { Extraordinary flood - approx. 20 000 } \\
\text { persons }\end{array}$ & 383,616 \\
\hline $\begin{array}{l}\text { Leakage of a dangerous chemical } \\
\text { substance }\end{array}$ & 416,7 \\
\hline Ice arena - 4 000 persons & 510,3 \\
\hline $\begin{array}{l}\text { Pivovar Janáček, the brewery - 2 000 } \\
\text { persons }\end{array}$ & 369,9 \\
\hline $\begin{array}{l}\text { Raciola Jehlička, the company - 2 000 } \\
\text { persons }\end{array}$ & 369,9 \\
\hline Traffic accident & 88,92 \\
\hline Railway accident & 156 \\
\hline Fire & 213,49 \\
\hline Zbrojovka, the company & 37,26 \\
\hline Rumpold, the company & 41,6 \\
\hline $\begin{array}{l}\text { RPG Recycling, , the company - 15 000 } \\
\text { persons }\end{array}$ & 561,6 \\
\hline Windstorm & 16,884 \\
\hline Forest fire & 24,12 \\
\hline
\end{tabular}

Table 1 The resulting level of risk for types of hazards occurring in the town of Uherský Brod

The numeric value of the risk level established for individual types of emergency events has a weighting factor of a comparative coefficient in the process of risk cumulation. [1]

\subsubsection{The Hazard Map}

Creating a hazard map is the first phase of the risk mapping. Its basis is the maps of individual types of hazards, or depicted manifestations of particular emergency events. [1]

The hazard map is created by merging maps of individual types of hazards. 


\section{Legend}

Ammonia, Ice arena $320 \mathrm{~m}$ Ammonia, Ice area $480 \mathrm{~m}$

- Ice area

Q5 Uherský Brod

Q20 Uherský Brod

Propane, Zbrojovka 204m

Propane, Zbrojovka $100 \mathrm{~m}$

- Zbrojovka, the company

Ammonia, Nipos $59 \mathrm{~m}$

Ammonia, Nipos $23 \mathrm{~m}$

- Nipos, the company

Chlorine, Delfin 292m

Chlorine, Delfin $172 \mathrm{~m}$

- Aquapark Delfín

Ammonia, Raciola 438m

Ammonia, Raciola $204 \mathrm{~m}$

- Raciola Jehlička, the company

Ammonia, Janáček $219 \mathrm{~m}$

Ammonia, Janáček $104 \mathrm{~m}$

- Pivovar Janáček, the brewery

Q100 Uherský Brod

Special flood

Ammonia, rail transport $286 \mathrm{~m}$

-. Railway

Protective arrea for bird flu $3 \mathrm{~km}$

Outbreak of swine flu birds $100 \mathrm{~m}$

- Zemaspol, the company

- Petroll, road transport $132 \mathrm{~m}$

- Road

Forest areas

Snowy areas

0.2

ए 0.3

| $\quad 0.4$

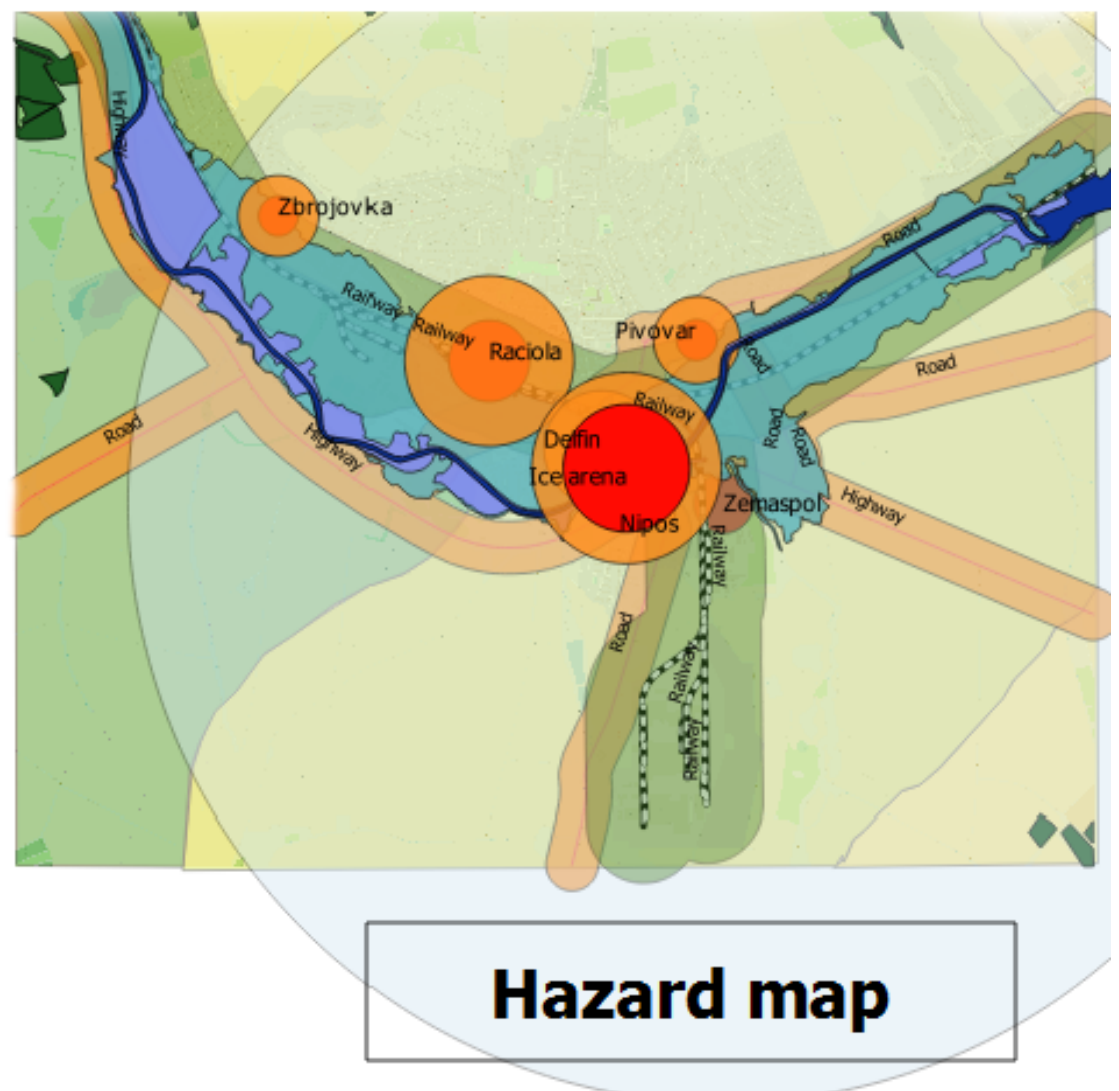

Fig 1 Hazard map

\subsubsection{The Vulnerability Map}

The second phase of risk mapping includes the creation of a vulnerability map. The vulnerability of a territory can be understood as the susceptibility of the territory to the effects of an emergency event. For the purposes of risk mapping, the cumulative vulnerability indicator, which is determined by merging the vulnerability sub-elements, is used to express the level of possible loss and damage in the analyzed territory. [4]

The vulnerability is cumulated in the area where individual elements of vulnerability intersect. The individual elements of vulnerability include population (population density), crucial and public infrastructure and the environment. 


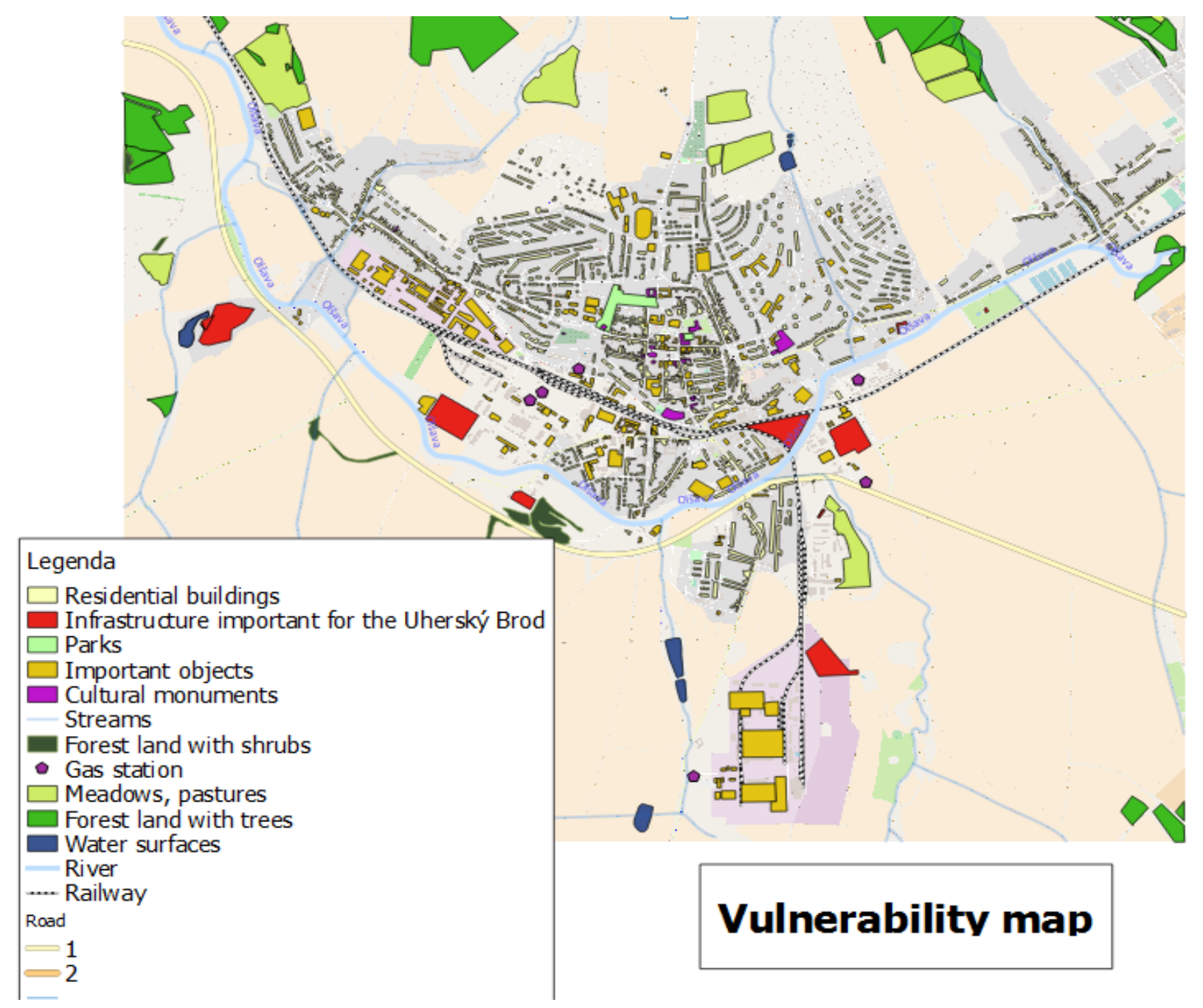

Fig 2 Vulnerability map

For the purposes of risk mapping, the so-called polygon layers are used; the polygons are overlapped. The vast majority of the said vulnerabilities are represented by point or line layers in cartographic expression and therefore these layers need to be converted to polygons. In risk mapping this is solved by means of the so-called buffers of a given radius. [9]

\subsubsection{The Map of Cumulative Risk}

Creating a cumulative risk map is the third phase of risk mapping. During this phase the vulnerability and hazard maps are merged.

The risk maps depict the level of risk in the town of Uherský Brod by means of colors.
The level of risk is expressed by a multilevel color scale, e.g.:

white color - zero risk,

green color - low risk

yellow color - medium risk,

orange color - high risk.

The area with the highest risk should be further investigated, primarily in order to eliminate this risk. 


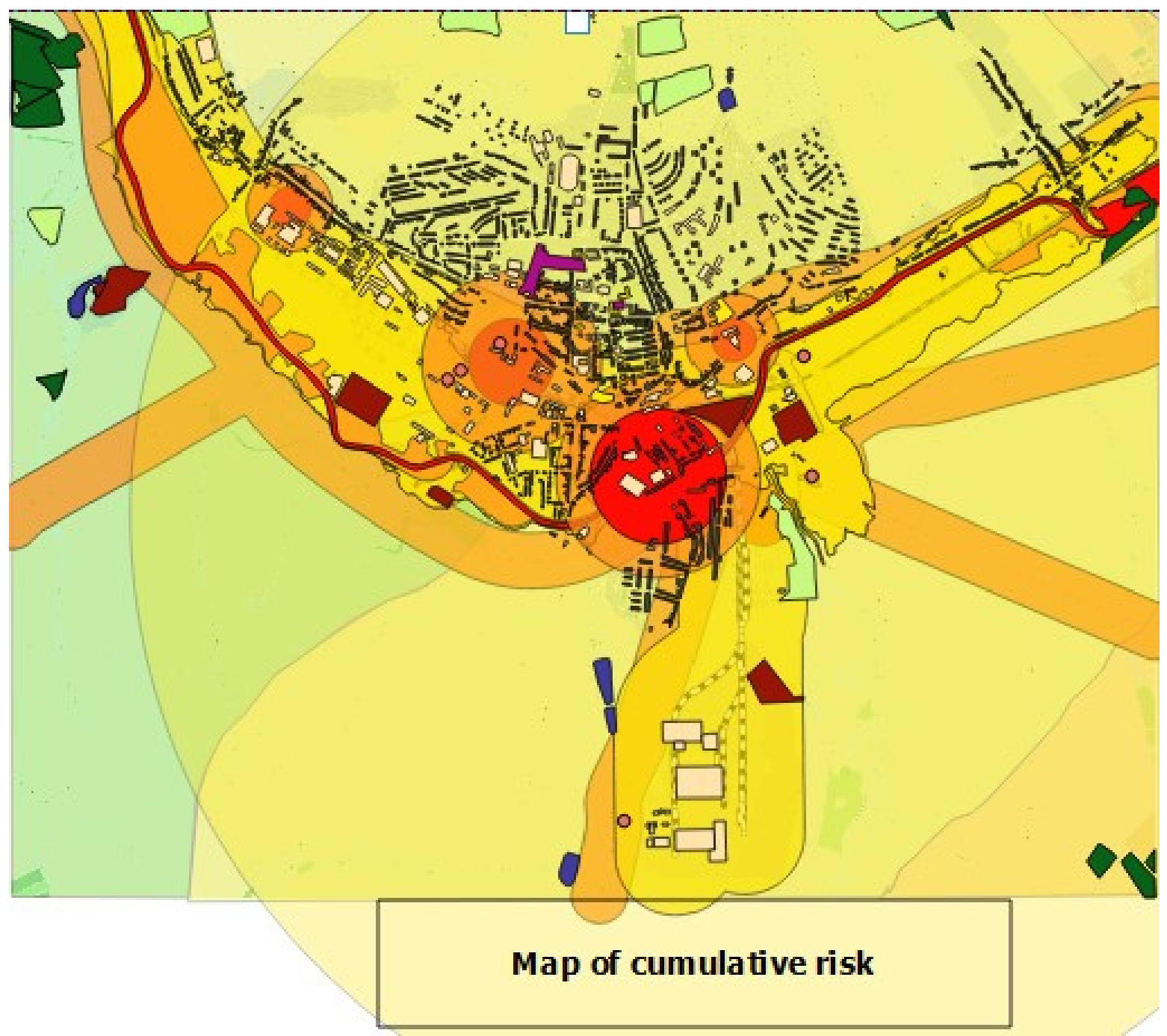

Fig 3 Map of cumulative risk

The main output of the article is the interaction of hazards and vulnerability, which leads to the determination of cumulative risk. In the resulting map it is possible to see where the cumulative risk is imminent and where it is not. It is also possible to estimate that the most serious emergency event in the town of Uherský Brod is a hundred-year flood. In the event of overflowing of such an amount of water, secondary emergency events may be expected (e.g. leakage of chlorine from a cooling device, etc.).

The results of risk mapping are presented on special maps that visually serve for better understanding of interconnections among individual types of risk and which allow the identification of the composition and level of risk for the given territory. They contain comprehensive information on levels of risk in the area and others. In general, they show which parts of the town of Uherský Brod are at the highest risk level and thus, they could serve as one of the information elements for the population of the town. Depending on the results, it is possible to create a manual for the inhabitants, which would include a map with a legend, information on the highest risks in the town and basic instructions on how to act during the EE.

These maps also represent a basic input into emergency and crisis planning processes. They can serve as a basis for the design of improvised shelters or for dislocation of warning and information elements depending on the risk areas. Another advantage is the possibility of planning routes for rescue and salvage operations (population evacuation, transportation of supplies, etc.). [2][4]

\section{Conclusion}

Risk mapping involves five phases. It should be noted that these phases were limited in the article, 
and only the first three phases were addressed, these three phases are the hazard map, the vulnerability map and the cumulative risk map. In the first part of the risk mapping, the sources of hazards were mapped within the analyzed territory. In particular, these included transport roads, railway transport, entities handling dangerous chemical substances and rivers (simulations of twenty-year, fifty-year and hundred-year floods). In addition, significant and vulnerable objects were mapped to represent the vulnerability of the area. These included roads, crucial infrastructure, railways, power lines, cultural monuments and other important objects, biotic environment and, above all, the population. By means of all this the hazard map and the vulnerability map were created. The interaction of these two maps then led to the map the cumulative risk.

The resulting risk map showed where hazards can cumulate in the analyzed territory of the town of Uherský Brod and where these hazards can negatively affect the individual vulnerabilities. The area with the highest risk level should be further investigated, primarily in order to eliminate the risk.

In addition, publishing the resulting risk maps seems to be effective as well; this could serve for an efficient notification to the civilians about hazards that exist in the territory of the town of Uherský Brod. Moreover, a primary emphasis could then be placed on education of the population in the field of population protection, or on the principles of appropriate behavior during an emergency event.

The major disadvantage of risk mapping is the impossibility of including hazards that cannot be easily expressed in space because their manifestations within the town of Uherský Brod are difficult to predict, e.g. earthquakes, meteorite fall, fire of natural origin or economic instability. These phenomena cannot be included into risk mapping.

Other limitations include designation of different levels of risk because it is difficult to segregate them. This designation depends on the person who evaluates the risks. What is perceived as medium risk by one person can be perceived as high risk by another. However, at the conclusion of the mapping the emphasis is placed on the value of the level of risk; the representation on the map in the color scale is understood only as a visualization of the results.

Thanks to the application of the risk mapping method in the town of Uherský Brod the initial presumption of the suitability of this method for the analysis of safety risks was confirmed.
A high degree of visualization associated with the use of GIS tools can be perceived as a significantly positive outcome. Therefore, the outputs are more legible and clear and allow easy presentation of results to both professionals and the population.

In conclusion, there is a potential for further elaboration of the results, especially for the implementation of the final steps of the risk mapping method, which are: determination of preparedness and amended risk. These parts were limited due to their complexity. For the verification of applicability of the method is the resulting risk mapping sufficient.

The implementation of these parts is a subject of further research. Similarly, the preparation of educational materials for increasing the preparedness of the population will be the focus of future research. This step is directly related to one of the final stages of risk mapping, which is determining the preparedness.

Acknowledgements. This paper is supported by the Internal Grant Agency at Tomas Bata University in Zlín, projects No. IGA/FLKR/2017/003; and No IGA/FLKŘ/2018/001.

\section{References:}

[1] Kromer A., Musial P., Folwarczny L.: Mapování rizik, Spektrum (Sdružení požárního a bezpečnostního inženýrství), pp. 16-66, 2010, Ostrava, 126 s. ISBN 978-807385-086-9. (2010)

[2] A. ZABRANSKA, J. RAK, P. SVOBODA. Risk Mapping in the town of Uherský Brod In: Applied Physics, System Science and Computers III: Proceedings of the 3nd International Conference on Applied Physics, System Science and Computers (APSAC2018), September 25-28, 2018, Dubrovnik, Croatia, 2018. Springer. ISSN 978-3-31975605-9.

[3] Brehovsky M., Jedlička K.: Úvod do geografických informačnich systémů, pp. 1126, Plzeň, University in South Bohemia, Avaible from: http://gis.czu.cz/studium/ugi/eskripta/ugi.pdf. (2010).

[4] QGIS - The Leading Open Source Desktop GIS [online] Available from: https://www.qgis.org/en/site/about/index.html (2018).

[5] Balint, T.: Aplikace geografických informačnich systému $v$ oblasti ukrytí obyvatelstva. J. Bachelor thesis.: Tomas Bata 
University in Zlín, FLCM, Czech Republic, (2018)

[6] GIScom: Geoinformation solutions [online] [cit. 2018-09-24]. Available from: http://www.giscom.cz/en. (2010)

[7] GIS Understanding spatial media. First published. Editors Rob KITCHIN, Tracey P. LAURIAULT and Matthew W. WILSON. Los Angeles ; London ; New Delhi ;, Singapore ; Washington DC ; Melbourne: Sage, (2017)
[8] NÉTEK, Rostislav a Tomáš BURIAN. Free and open source $v$ geoinformatice. First published. Olomouc: Palacký University (2018)

[9] ZTOMASZEWSKI, Brian. Geographic information systems (GIS) for disaster management. Boca Raton: Springer Berlin Heidelberg, 2015. ISBN 978-148-2211-689. 